\title{
An Improvement to Advanced Centroid Location Algorithm
}

\author{
Nana Gong ${ }^{1, a}$, Haiyan $\mathrm{Wu}^{1, b}$ \\ ${ }^{1}$ School of Information and Engineering, Huanghe Science and Technology College, Zhengzhou, \\ 450063, China \\ aemail,: gongna163@163.com, bemail: 775959406@qq.com
}

Keywords: wireless sensor networks, unknown nodes, beacon nodes, centroid location algorithm

\begin{abstract}
On the basis of ACLA(Advanced Centroid Location Algorithm), IACLA (Improvement to Advanced Centroid Location Algorithm) was proposed. IACLA used the located unknown nodes and beacon nodes to get the final estimate. It was equivalent to weighted centroid location algorithm, and made the weights more reasonable. Analysis and simulation results show that IACLA produces smaller error than ACLA with the same coverage rate of location.
\end{abstract}

\section{Introduction}

Wireless sensor networks extend our ability to monitor and control the physical world. For special applications of wireless sensor networks, locations of nodes are generally random and unknown. In large sensor network systems, we need nodes to be able to locate themselves. For example, localization is of great importance to track an object moving through the network. Many localization algorithms for sensor networks have been proposed to provide per-node location information. With regard to the mechanisms of location, schemes are divided into two categories: range-based and range-free. Because of the hardware limitations and low energy of WSN devices, solutions in range-free localization are a cost-effective alternative to range-based approaches which are more expensive.

Some range-free algorithms have been presented by scholars, such as CLA(Centroid Location algorithm)[1], APIT[2], DV-HOP[3], amorphous positioning[4] and bounding box[5]. Centroid location is a range-free and coarse-grained algorithm for outdoor. Beacon nodes broadcast their positions to neighbours within single hop. A sensor node computes the centroid using all received beacon messages. It has been improved by making an adjustment in the distribution or taking the weighted average of beacon nodes[6]. APIT is a range-free localization scheme for large scale sensor networks. The perfect P.I.T test is infeasible in practice; however, we can still use an approximation method APIT to obtain a very high level of accuracy. In DV-HOP, beacon locations are flooded through the network and the running hop counts are kept, so the average one hop distance is estimated. Amorphous positioning is similar to DV-HOP but uses communication radius instead. A discrete model of a random network is presented in bounding box algorithm. It needs much less computing power to calculate the intersection of two squares than to compute the intersection of two discs.

The article makes the following contributions:

- It analyses the theory of advanced centroid location algorithm and points out its irrationality.

- It proposes an improvement to advanced centroid location algorithm, and demonstrates its validity.

\section{ACLA}

Coverage rate and accuracy of location are the performance people are more concerned about. Coverage rate is the proportion of nodes which can locate themselves. We usually characterize the location precision by AE(Absolute Error) and RE(Relative Error).We define a few terms:

$N$ : Total number of unknown nodes

$N_{1}$ : Number of located unknown nodes 
$\left(X_{\text {est }}, Y_{\text {est }}\right)$ : Estimated location of unknown node

$\left(X_{a}, Y_{a}\right)$ : Actual location of the unknown node

$R$ : Transmission range of the node

We characterize the coverage rate by $\mathrm{CR}$ defined as equation 1.AE and RE are shown in equation 2 and 3 . When the RE is less than 0.4 , its impact on the performance of routing and target tracking is not obvious.

$$
\begin{gathered}
C R=\frac{N_{1}}{N} \\
A E=\sqrt{\left(X_{\text {est }}-X_{\mathrm{a}}\right)^{2}+\left(Y_{\text {est }}-Y_{\mathrm{a}}\right)^{2}} \\
R E=\frac{\sqrt{\left(X_{\text {est }}-X_{\mathrm{a}}\right)^{2}+\left(Y_{\text {est }}-Y_{\mathrm{a}}\right)^{2}}}{R}
\end{gathered}
$$

In CLA, unknown node which has no beacon nodes in its communication region can't be located. So the coverage rate of location is too low to meet the requirements in application. ACLA for wireless sensor network is presented[7]. The localization algorithm goes as follows.

Step 1 The initial estimate:For each unknown node, finds out all the beacons, and localizes itself as the centroid of the polygon composed of above beacons. It can be shown that

$$
\left(X_{\text {est }}, Y_{\text {est }}\right)=\left(\frac{X_{1}+\cdots+X_{k}}{k}, \frac{Y_{1}+\cdots+Y_{k}}{k}\right)
$$

$k$ : Number of connected beacons

$\left(X_{1}, Y_{1}\right), \ldots,\left(X_{\mathrm{k}}, Y_{\mathrm{k})}\right.$ Locations of connected beacons

Step 2 The final estimate: For each unknown node, uses the initial estimates in its communication region instead of the beacons to obtain the final estimate.

The advantages of ACAL are

(1) The unknown nodes which can't be located initially can be located by the initial estimates. So the coverage rate of location increases.

(2) Using the initial estimates which are determined by the beacons outside the region is equivalent to using more beacons. To some extent, it will improve the localization precision.

The simulation results indicate that the ACLA has an obvious improvement in localization precision than CLA.

\section{IACLA}

It should be noted that some location errors will increase sometimes in ACLA. This is illustrated in Fig.1. Beacon nodes $b_{1}$ and $b_{2}$ are in the communication range of unknown node $N_{1}$, and beacon nodes $b_{2}$ and $b_{3}$ are in the communication range of unknown node $N_{2}$. 


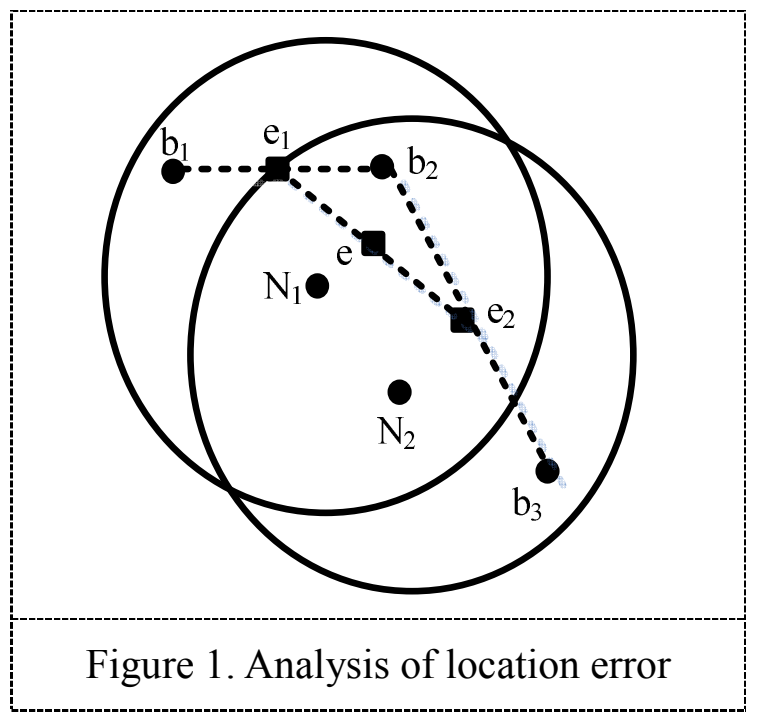

In ACLA, initial estimated location of $N_{1}$ is

Initial estimated location of $N_{2}$ is

$$
e_{1}=\frac{b_{1}+b_{2}}{2}
$$

$$
e_{2}=\frac{b_{2}+b_{3}}{2}
$$

The final estimated locations of $N_{1}$ and $N_{2}$ are

$$
e=\frac{e_{1}+e_{2}}{2}=\frac{\frac{b_{1}+b_{2}}{2}+\frac{b_{2}+b_{3}}{2}}{2}=\frac{1}{4} b_{1}+\frac{1}{2} b_{2}+\frac{1}{4} b_{3}=\sum_{i=1}^{3} w_{i} b_{i}
$$

Compared with $e_{1}$ and $e_{2}$, error of $N_{1}$ decreases while error of $N_{2}$ increases. We analyze the weights

$$
w_{1}=\frac{1}{4}, w_{2}=\frac{1}{2}, w_{3}=\frac{1}{4}
$$

For $b_{3}$ is farther from $N_{1}$ than $b_{1}$, the $w_{3}$ should be smaller than $w_{1}$.It is unreasonable that $w_{3}$ is equal to $w_{1}$ in ACLA.

Improvement: We use not only the located unknown nodes but also the beacon nodes for the final estimate. Estimated location of $N_{1}$ is

$$
\begin{gathered}
e=\frac{b_{1}+b_{2}+e_{1}+e_{2}}{4}=\frac{b_{1}+b_{2}+\frac{b_{1}+b_{2}}{2}+\frac{b_{2}+b_{3}}{2}}{4}=\frac{3}{8} b_{1}+\frac{1}{2} b_{2}+\frac{1}{8} b_{3} \\
w_{1}=\frac{3}{8}, w_{2}=\frac{1}{2}, w_{3}=\frac{1}{8}
\end{gathered}
$$

It is reasonable that $w_{1}$ is larger than $w_{3}$. This is the main idea of improvement to advanced centroid location algorithm, which we call IACLA. The localization algorithm goes as follows.

Step 1 The initial estimate $\left(X_{e s t}, Y_{e s t}\right)$ :For each unknown node, finds out all the beacons, localizes itself as the centroid of the polygon composed of above beacons via equation 4 and notes the number of connected beacons $k$.

Step 2 The final estimate $\left(X^{\prime}, Y^{\prime}\right)$ : For each unknown node, uses $m$ initial estimates and $k$ beacon nodes in its communication region to obtain the final estimate via equation 5 .

$$
\left(X^{\prime}, Y^{\prime}\right)=\left(\frac{k X_{e s t}+X_{e s t 1}+\cdots+X_{e s t m}}{k+m}, \frac{k Y_{e s t}+Y_{e s t 1}+\cdots+Y_{e s t m}}{k+m}\right)
$$

Now we analyze the CR and amount of computation of IACLA compared with ACLA. 
(1) CR :The difference between IACLA and ACLA is in step 2, and it doesn't impact the CR. So CR of IACLA is as same as ACLA.

(2) Amount of computation: To note the number of beacon nodes $k$ doesn't increase the amount of computation.

\section{Simulation results}

We simulate ACLA and IACLA, with randomly generated topologies of 100 unknown nodes and 36 beacon nodes in a $100 m \times 100 m$ square region (Fig.2 and Fig.3). The meanings of symbols:

$*$ :Beacon node

$\mathrm{x}$ :Unknown node which can't be located

o: Actual location

+ : Estimated location

From this concrete example, we can see IACLA has the same CR but smaller error than ACLA.
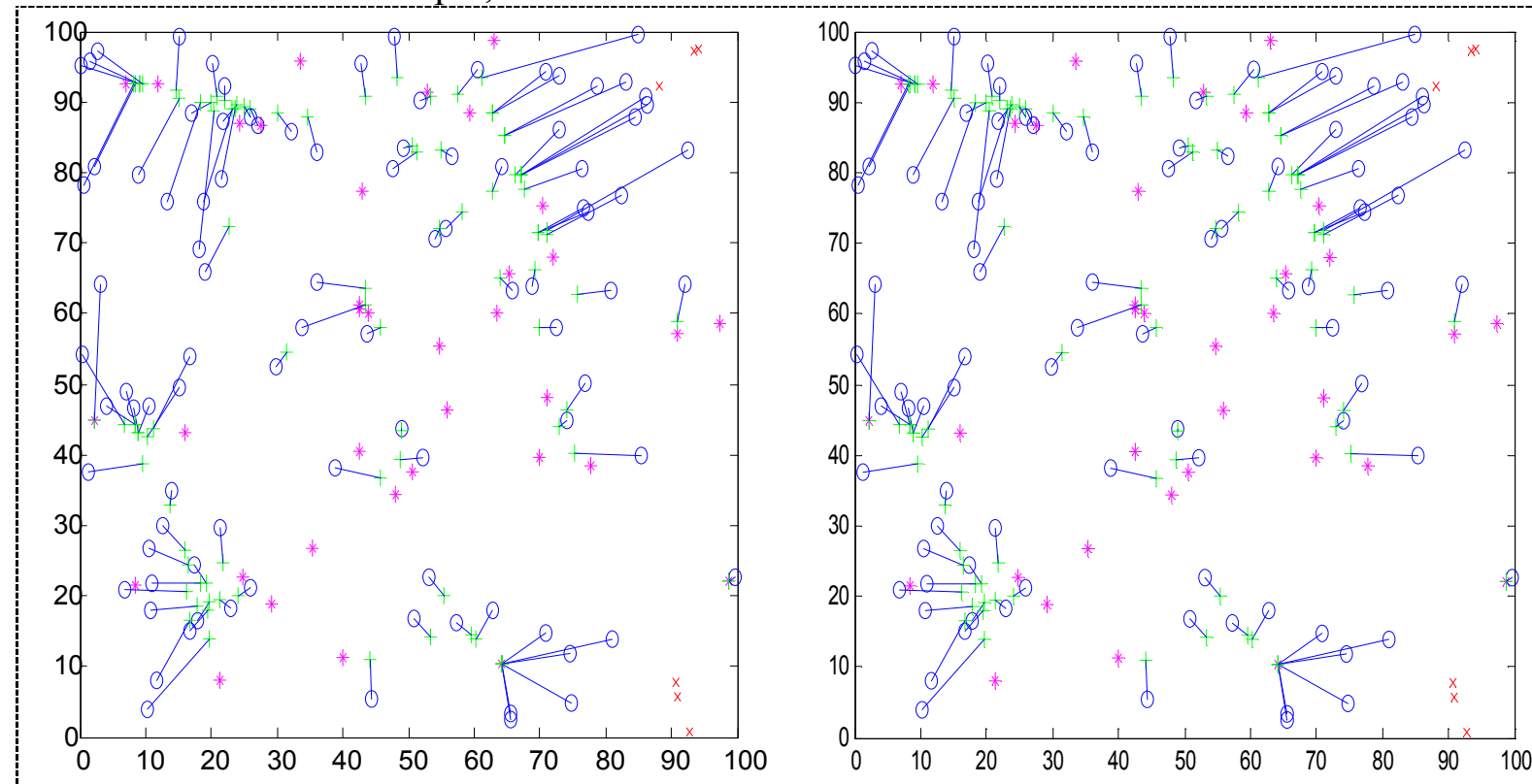

Figure 2. $\mathrm{ACLA}(\mathrm{CR}=0.94, \mathrm{AE}=8.05, \mathrm{RE}=0.54)$

Figure 3. IACLA $(\mathrm{CR}=0.94, \mathrm{AE}=7.74, \mathrm{RE}=0.52)$

In order to removal the randomness, we do 10000 experiments to get the average results: $\mathrm{CR}, \mathrm{AE}$ and RE. Compared with ACLA, IACLA produces the same CR but lower AE and RE with incresing radio range (Fig.4, Fig.5 and Fig.6).It has an obvious improvement when the radio range is from $30 \mathrm{~m}$ to $90 \mathrm{~m}$.

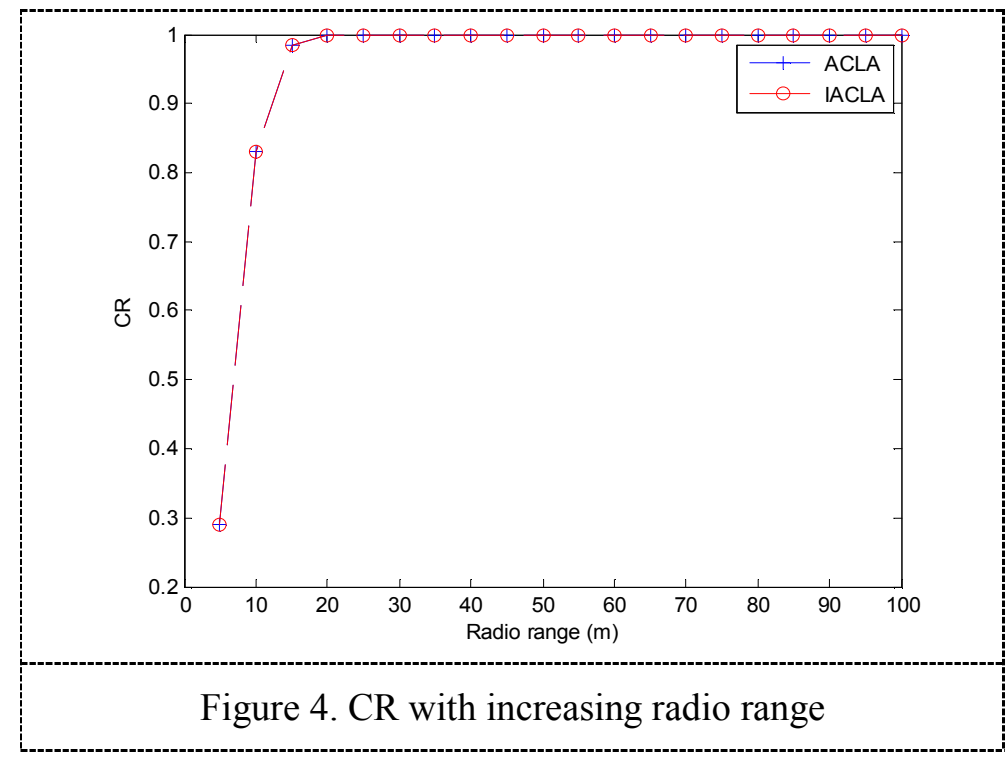




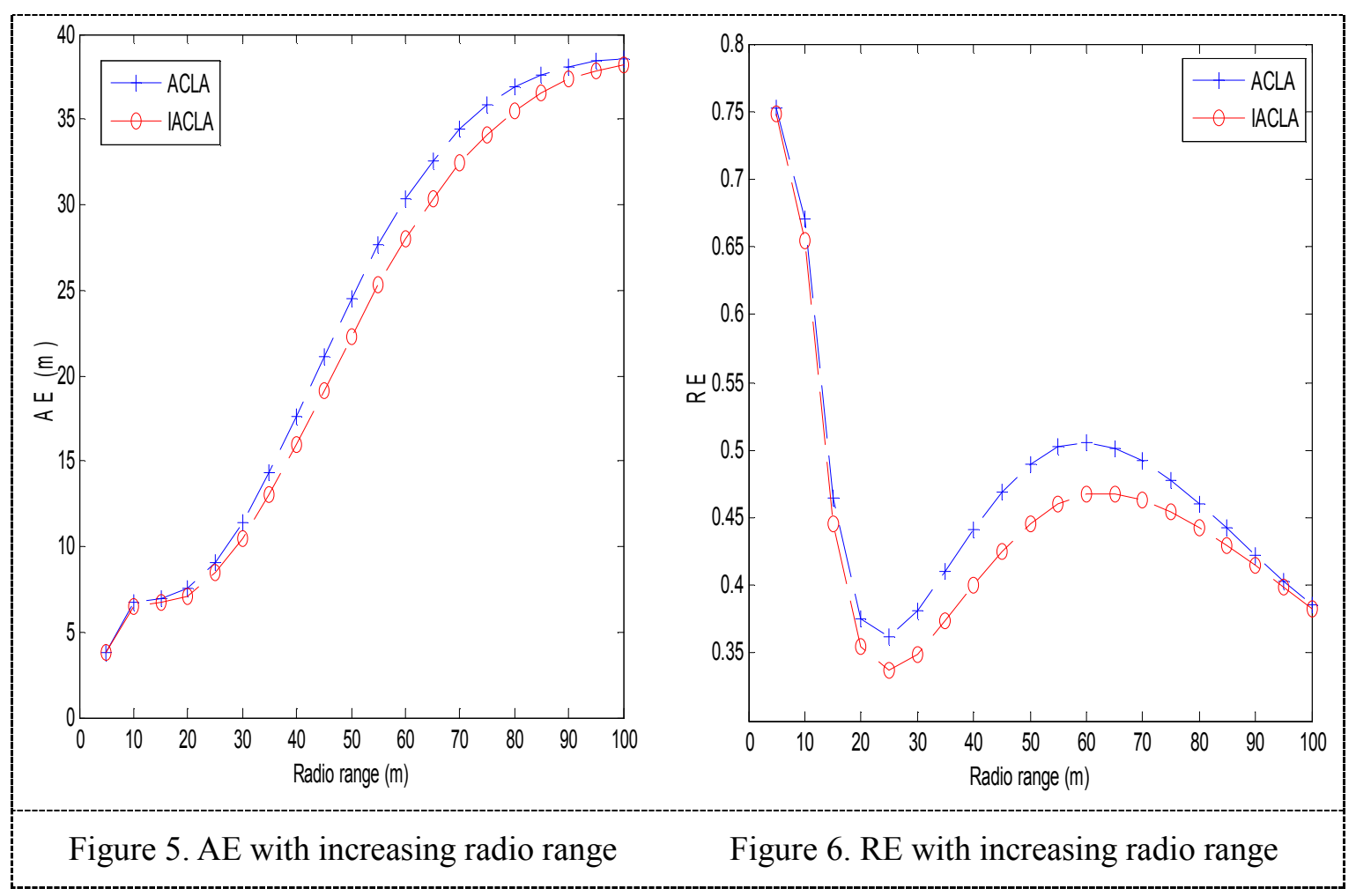

For $\mathrm{CR}$ is obviously same, and $\mathrm{AE}$ divided by radio range equals $\mathrm{RE}$. So $\mathrm{AE}$ can indicate the error under the same condition. We give AE results shown in Fig.7 and Fig.8.It is obvious that IACLA produces smaller error than ACLA. When the number of beacon nodes increases, the AE decreases(Fig.7). The reason is that locations of beacon nodes are more accurate than estimates. Because of the cumulative errors, when the number of unknown nodes increases, the $\mathrm{AE}$ increases(Fig. 8).
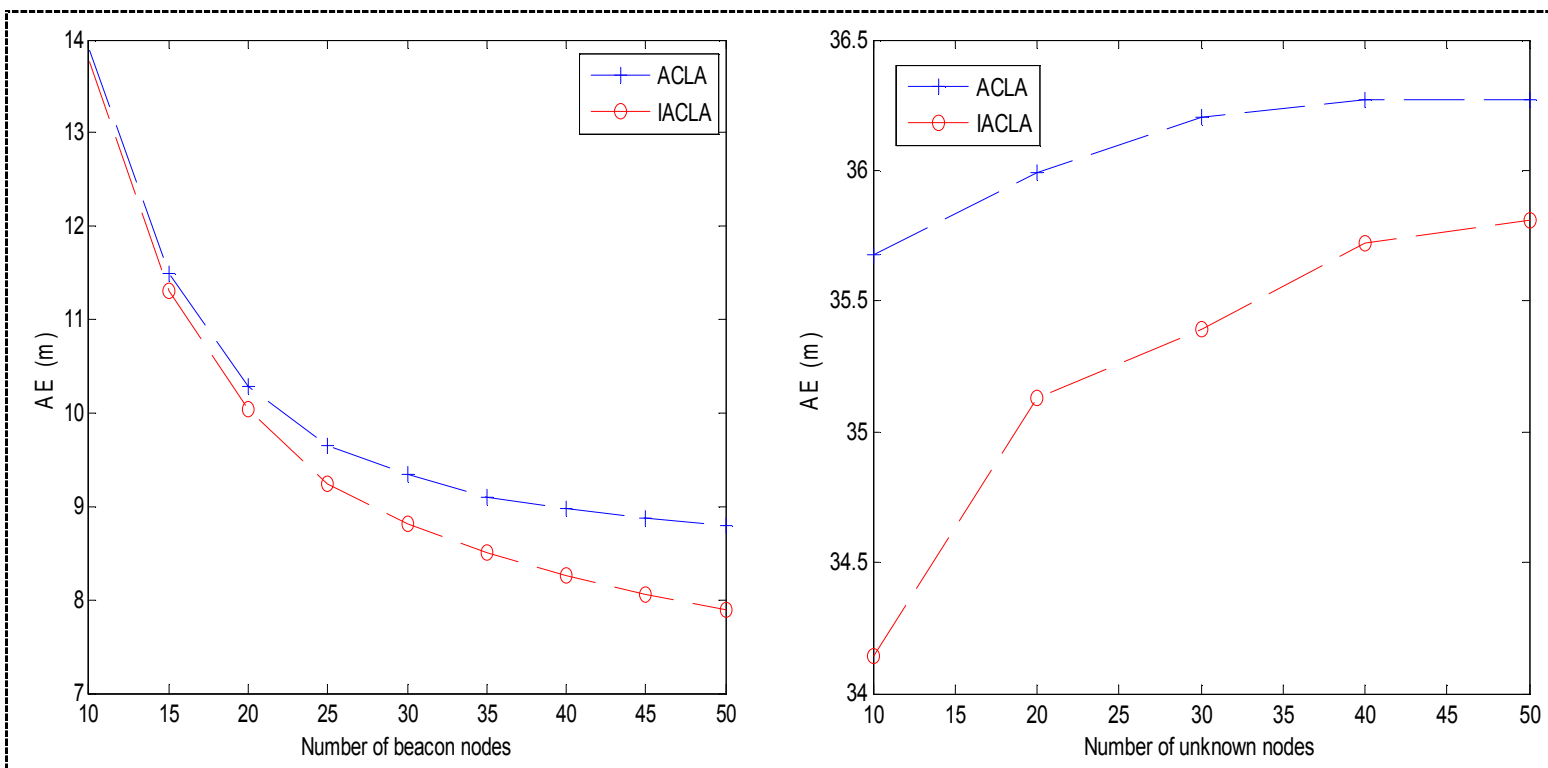

Figure 7. AE with increasing number of beacon nodes $($ radio range $=25$ )

Figure 8. AE with increasing number of unknown nodes $($ radio range $=70$ ) 


\section{Conclusion and future work}

The main contributions of this paper are a range-free algorithm IACLA and estimates of its coverage rate and error for localization of nodes in wireless sensor network. This is just one of the improvements to ACLA. There are some others improvements. For example, if all the nodes are used for the estimate, error may increase sometimes. So which nodes should be used for the estimate is worthy of deep-going study. We plan to address these issues in future work.

\section{Acknowledgments}

This reach is supported by Science and Technology Innovation Team of IOT in Zhengzhou under grant 112PCXTD343, and major research projects in Henan Province Science and Technology Agency under grant 112102310582.

\section{References}

[1] Bulusu N,Heidemann J,Estrin D.GPS less low cost outdoor location for very small devise[J].IEEE Personal Communications Magazine, 2000, 7(5): 28-34.

[2] He T,Huang C,Blum B M,Stankovic J A,Abdelzaher T.Rangr-free localization schemes for large scale sensor networks[C].San Diego,California,USA:ACM MobiCom,2003:81-95.

[3] Niculescu D, Nath B. DV based positioning in ad hoc networks[J]. Journal of Telecommunication Systems, 2003,22(1/4):267-280.

[4] Nagpal R, Shrobe H, Bachrach J. Organizing a global coordinate system from local information on an ad hoc sensor network. In: Zhao F, Guibas LJ, eds. Proc. of the 2nd Int'l Workshop on Information Processing in Sensor Networks (IPSN). New York: Springer-Verlag, 2003. 151152.

[5] Simic S N, Sastry S. Distributed localization in wireless ad hoc networks[M].U C Berkeley,2002,UCB-ERL-M02-26.

[6] Bulusu B, Heidemann J,Estrin D. Density adaptive algorithms for beacon placement in wireless sensor networks[C].In:IEEE ICDCS'01, Phoenix,AZ,April 2001.

[7] LI Zhao-bin, WEI Zhan-zhen, XU Feng-li.The Performance Analysis of Advanced Centroid Localization Algorithm for Wireless Sensor Network[J]. Chinese Journal of Sensors and Actuators. 2009, 22(4):563-566. 